\title{
Komplikationen verhindern
}

\section{CED bei Kindern: Welche Therapie ist am besten?}

Kinder sind keine kleinen Erwachsenen. Das gilt auch für die Therapie chronisch entzündlicher Darmerkrankungen; denn eine Langzeittherapie mit Kortikosteroiden führt zur Wachstumsretardierung.

„Die Hausärzte sind die Kinderärzte von morgen; denn die Pädiater sind eine aussterbende Spezies“, so Prof. Michael Radke, Rostock. Dies müsse bei der Ausund Weiterbildung der Hausärzte stärker berücksichtigt werden. Neben der Akutversorgung bei Infektionen und Unfällen seien psychische Störungen eine zunehmende Herausforderung. Dazu kommen chronische Erkrankungen und Malignome, die nach den Unfällen die zweithäufigste Todesursache bei Kindern darstellen.

\section{Von A wie Asthma bis Z wie Zöliakie}

Etwa 10\% aller Kinder erkranken bis zum 18. Geburtstag an einer chronischen Erkrankung. „Chronische Krankheiten bei Kindern sind immer ein Störfaktor einer normalen Entwicklung“, so Radke. Wenn der frühe Krankheitsbeginn in die Phase der größten Wachstumsgeschwindigkeit falle, würden Entwicklungsprozesse verlangsamt und kompliziert. Gerade die Pubertät sei eine Lebensphase, die durch die Entwicklung der eigenen Identität und des Selbstbildes, die Unabhängigkeit von den Eltern, die kognitive Entwicklung, die Berufswahl und die Entwicklung der Sexualität geprägt sei.

Das Spektrum der chronischen Erkrankungen bei Kindern ist breit. Es reicht von A wie Asthma bronchiale bis $\mathrm{Z}$ wie Zöliakie. Dazu gehören das Asthma bronchiale, der Diabetes mellitus Typ 1, Epilepsien, Mukoviszidose, Nierenkrankheiten, Malignome und chronisch entzündliche Darmerkrankungen, nämlich Morbus Crohn und Colitis ulcerosa.

„Die Inzidenz der chronisch entzündlichen Darmerkrankungen hat gerade auch bei Kindern in den letzten Jahrzehnten deutlich zugenommen“, so Radke. Dies hänge mit den veränderten Lebens- und Ernährungsbedingungen zu- sammen. Die Erkrankungen können schon im ersten Lebensjahr auftreten, ein erster Häufigkeitsgipfel zeigt sich in einem Alter zwischen 10 und 14 Jahren. Beim Morbus Crohn ist auch häufiger als bei Erwachsenen der gesamte MagenDarm-Trakt betroffen. Wichtig in dieser Altersgruppe ist, dass der Entzündungsprozess früh, rasch und effektiv bekämpft wird, um einen langanhaltenden Krankheitsverlauf mit daraus resultierenden Komplikationen wie Fisteln oder Strikturen zu verhindern. „Je länger die Entzündung anhält, umso höher ist Risiko für Komplikationen wie Strikturen und auch das Risiko für einen operativen Eingriff steigt“, so Radke. Gerade bei Kindern müssten diese Erkrankungen extensiver und aggressiver therapiert werden.

\section{Optimale Lebensqualität erreichen}

Ziel der Therapie bei Kindern mit CED ist die Herstellung einer anabolen Ernährungssituation, die Vermeidung dauerhafter Wachstumsdefizite und wachstumsge- fährdender Osteopathien. Dadurch soll auch eine adäquate Pubertätsentwicklung und optimale Lebensqualität erreicht werden. Eine Langzeittherapie mit Kortikosteroiden ist bei Kindern im Hinblick auf das Längenwachstum sehr problematisch. Deshalb besteht die Notwendigkeit, Immunsuppressiva und auch Biologika wie Tumornekrose-Faktor- $\alpha$-Inhibitoren frühzeitig einzusetzen.

\section{Therapieziel: Mukosaheilung}

Aber bei CED geht es sowohl bei Kindern als auch bei Erwachsenen nicht nur darum, dass der Patient beschwerdefrei wird. „Das eigentliche Therapieziel sollte die Mukosaheilung sein, weil eine solche für den weiteren Krankheitsverlauf günstig ist, also mit weniger Komplikationen assoziiert ist“, so Radke. Ein solches „mucosal healing“ könne mit einem Biologikum sehr viel eher erreicht werden. Auch konnte man in Studien zeigen, dass eine effektive Entzündungskontrolle das Aufholwachstum bei den betroffenen Kindern beschleunigt.

Dr. Peter Stiefelhagen

\section{Forum: Die Hausarztpraxis im Fokus, 12.5.2017 in München}

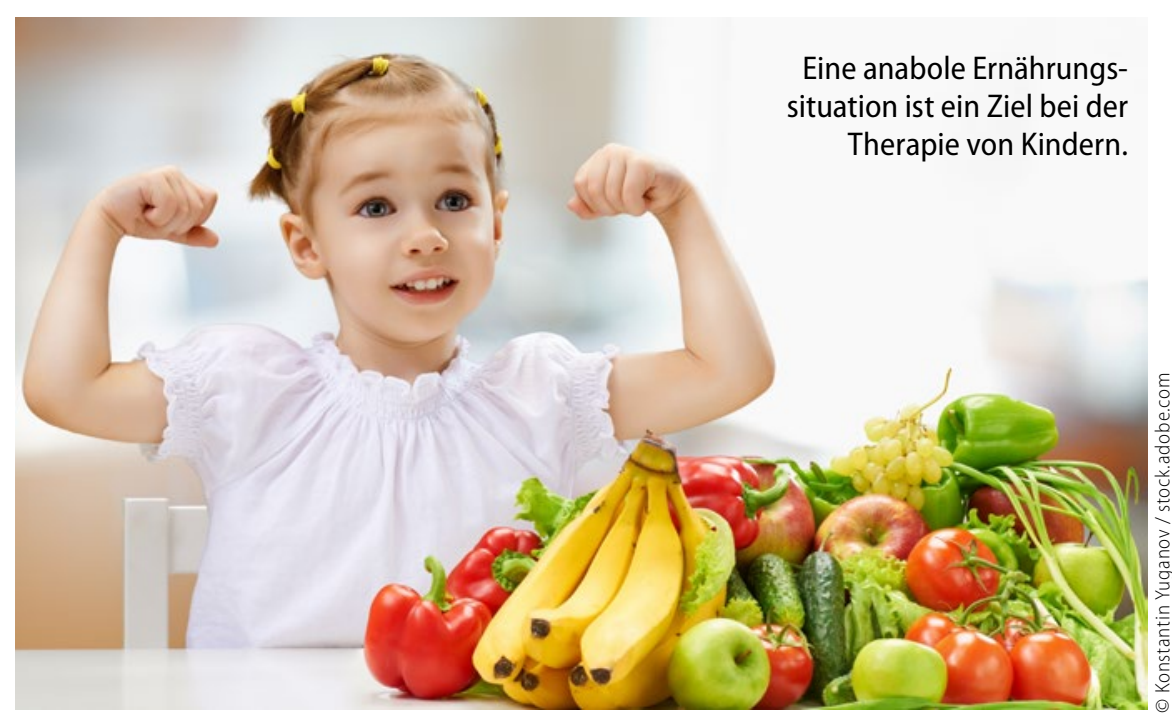

